\title{
Research ethics review: Do it once and do it well
}

$\mathrm{R}$ esearch ethics boards are essential for protecting the rights and well-being of research participants. Currently, however, investigators, research institutes and funders are complaining that the system does not work. A chief complaint is that multicentre trials are overreviewed and mired in bureaucracy because each centre performs its own independent reviews.

A simple calculation suggests that, for a 20-centre trial, some 300-500 person-hours in total may be required of the parties involved in the ethics review process. Given this significant opportunity cost, one would hope to find clear evidence that such a labour-intensive process protects patients effectively. Yet no such data exist. Getting input from a large number of committees may, in fact, adversely affect research participants by, for example, increasing the complexity of consent forms. ${ }^{1}$ Moreover, the need to conduct multiple independent reviews diminishes the time and expertise that can be allocated to the review and oversight of other studies. Time is also taken away from patient care, research and other valuable health care activities, in exchange for no proven benefit.

In North America, the ethics review process is generally dependent on local reviews, which are thought to be essential to ensure that ethical decisions reflect local conditions and culture. Although local conditions are important in multicentre trials, issues of local culture are less so. Costs and resource constraints in health care mean that research ethics boards are increasingly being asked "Do we have appropriate expertise and resources locally to undertake this study?" rather than "Is this study ethically sound?" More saliently, large national and international trials now often raise major issues related to liability, accountability and responsibility, as well as concerns about oversight once studies are underway. Although local research ethics boards can opine on these matters, without national guidance or the ability to speak as a collective, the voices of single committees often go unheard.

We need to overhaul the ethics review system from an autonomous local committee process into an interdependent collaboration of local committees working within a single national system. Although there are myriad rules and regulations governing research ethics in North America, all permit collaboration between ethics boards and would allow creation of a single decentralized review process. The new national system would replace totally independent multiple reviews with support of an interactive open review model at the local level. In such a redesign, we do not advocate that local institutions relinquish the right to approve studies; instead, we envision a streamlined and timely process that occurs locally once full ethics reviews have been conducted centrally. Our credo for research ethics review should become "do it once and do it well."

To initiate theses changes, an early priority should be the harmonization of institutional standards across the country.
Common forms could be developed to reduce the bureaucratic burden for multicentre trials. Once accepted standards are in place, institutions and ethics committees would be able to work together to review trials and share findings. Accountability would be further enhanced by adopting clear benchmarks to ensure timeliness and quality of reviews. The development of a national education program for new research ethics committee members and health researchers, as well as continuing education for more experienced members, could facilitate these changes and help streamline and enhance the function of all research ethics boards. Transparency, which is important for institutions that aim to protect the public and maintain public trust, would be served by posting all decisions on publicly accessible websites.

There has been some progress in Canada at the regional level. Quebec and Newfoundland and Labrador have adopted systems for centralized ethics review. The Ontario Cancer Research Ethics Board reviews cancer trials across that province. However, for improvements to occur across the entire system, we believe that a nationwide solution is both timely and necessary.

In Canada, none of this will happen without leadership from national institutions such as the Canadian Institutes of Health Research, the Panel on Research Ethics and other scientific councils. Leadership from the ethics and scientific communities will also be needed to build trust and a willingness to collaborate. Similar solutions should be sought in the United States. If all of Europe is envisioning collaborating on ethics reviews, there is no reason why we cannot do the same in Canada or even across North America.

\section{Paul Hébert MD MHSc \\ Editor-in-Chief \\ CMAJ \\ Raphael Saginur MD}

Chair, Governance Committee

Ontario Cancer Research Ethics Board

With the Editorial-Writing Team

(Matthew B. Stanbrook MD PhD, Ken Flegel MDCM MSc, Noni MacDonald MD MSc and Amir Attaran LLB DPhil)

Competing interests: See www.cmaj.ca/misc/edboard.shtml for the Editorial-Writing Team's statements. Paul Hébert is an employee of the Canadian Institutes of Health Research. The opinions expressed in this article are those of the authors and not of the Canadian Institutes of Health Research. Raphael Saginur is a coinvestigator of the Canadian Network for the Governance of Ethical Health Research Involving Humans, funded by the Canadian Institutes of Health Research.

\section{REFERENCE}

1. Burman W, Breese P, Weis S, et al.; Tuberculosis Trials Consortium. The effects of local review on informed consent documents from a multicenter clinical trials consortium. Control Clin Trials 2003;24:245-55. 